\title{
A practical guide to female sexual dysfunction: An evidence-based review for physicians in
} Canada

Yonah Krakowsky; Ethan D. Grober

Division of Urology, Department of Surgery, University of Toronto, Women's College Hospital \& Sinai Health System, Toronto, ON, Canada

Cite as: Can Urol Assoc J 2018 Feb. 23; Epub ahead of print. http://dx.doi.org/10.5489/cuaj.4907

Published online February 23, 2018

$* * *$

\section{Abstract}

Introduction: Female sexual dysfunction (FSD) is characterized as distress related to sexual pain, sexual arousal, sexual desire, and/or orgasmic dysfunction. Despite prevalence rates similar to male sexual dysfunction, women with sexual complaints have been long under-evaluated, treated, and studied. Over the last decade there have been advances in the medical evaluation and management of FSD, however, there remains a paucity of clinical resources available for women in Canada with sexual dysfunction.

Methods: The state of knowledge in the evaluation and treatment of FSD was reviewed. Recommendations are given for the practical evaluation and treatment of women with sexual symptoms that can be applied widely in Canada.

Results: Approaches to the management and treatment of FSD are discussed with a focus on the practical application of diagnostic and therapeutic tools in the management of sexual pain, low desire, low arousal, and orgasmic dysfunction.

Conclusions: There are evidence-based diagnostic and therapeutic approaches to FSD that can be broadly applied by Canadian physicians to improve access to female sexual medicine in Canada. 


\section{Introduction}

The evaluation and treatment of male sexual dysfunction has developed considerably since the release of sildenafil (Viagra ${ }^{\circledR}$ ) as a treatment for erectile dysfunction in $1998 .{ }^{1}$ There is a societal perception that it is important for men to preserve their sexual function and optimize their sexual performance. This perception, coupled with perpetual innovation in male sexual medicine, has led to many treatment options for male sexual dysfunction including oral therapies for erectile dysfunction, multiple vehicles for hormonal replacement, shockwave therapy, and penile implants for refractory erectile dysfunction. There are fellowships throughout Canada dedicated to the medical and surgical management of male sexual dysfunction. Medical students and residents across many disciplines are routinely exposed to the evaluation and treatment of men with sexual dysfunction. Unfortunately, despite robust clinical and academic interest in male sexual dysfunction, women with sexual complaints have been largely overlooked. ${ }^{2}$ There has been limited treatment options, few Canadian role models who specialize in female sexual medicine, and little academic activity in the area of female sexual function.

Fortunately, over the past decade there has been an increase in the clinical and academic interest in female sexual function. The times appear to be changing. The International Society for the Study of Women's Health (ISSWSH) was established in 2001 to serve as a multidisciplinary international community dedicated to advancing the study of female sexuality. There are published guidelines and position papers that reinforce the practical aspects of female sexual dysfunction (FSD) evaluation and management. ${ }^{3}$ There has been an important increase in research regarding the impact of cancer and its treatment on female sexual function. ${ }^{4}$ And finally, there are now FDA approved therapies for both low desire and sexual pain that will possibly be available in Canada in the future. ${ }^{5-7}$ The increase in attention to female sexual function over the past decade can be attributed to a number of factors including the establishment of ISSWSH, new treatments offering hope, an increase in female sexuality research, and broader societal forces promoting equity in medical practice and research.

This review provides a practical, evidence-based guide to the evaluation and management of FSD that is adaptable for clinical practice in Canada.

For the purpose of this review, the classification of FSD have been divided into four broad categories: sexual pain, low desire, low arousal and orgasmic dysfunction which closely mirrors the DSM V classifications of FSD, compromised of: genito-pelvic pain/penetration disorder (sexual pain), female sexual interest/arousal disorder (low desire and low arousal) and female orgasmic disorder (orgasmic dysfunction). ${ }^{8}$ These classifications have been chosen instead of the DSM classifications as many patients will not meet strict criteria but will still benefit from evaluation and management. A symptom based approach is the most effective means to organize the initial medical evaluation and treatment of women with sexual complaints to encourage collaboration and communication between healthcare providers. 


\section{Evaluation and treatment of female sexual dysfunction}

\section{Sexual pain}

\section{Evaluation}

Sexual pain is a common complaint in women of all ages and may include pain at the vulva, deep pain with penetration or tightening of the pelvic musculature. A complete medical history should include a gynecological history, medication review, history of birth control or hormonal therapy use, and a psychosocial history including a screen for mood disorders and abuse (table 1). Standardized sexual function survey such as the Female Sexual Function Inventory (FSFI) are an efficient way to assess patients at baseline and follow-up. ${ }^{9}$ Physical exam should include vulvoscopy if possible to document skin changes, specific points of sensitivity, labial measurements, assessment of the clitoris and clitoral hood, and a thorough assessment of the pelvic muscles with an internal exam. Vulvoscopy with high definition projected images allow patients to appreciate the anatomic changes that may be contributing to their symptoms. Cue tip testing at the vulva and vestibule may identify specific areas of tenderness that can be documented and reassessed at follow-up. Documenting baseline vaginal $\mathrm{pH}$ and followup values can determine if treatment has been effective as patients with atrophic vaginitis will often demonstrate elevated vaginal $\mathrm{pH}$ that will normalize with local hormonal therapy. Vulvar biopsy may be necessary to definitively diagnosis dermatological abnormalities (such as lichen sclerosis and lichen planus) as a cause of vulvar pain.

Patients who experience vulvar pain without an obviously identifiable cause (referred to as vulvodynia) can be further classified based on history and physical exam. Some patients may exhibit reproducible provoked pain (provoked vulvodynia) while others may have generalized vulvar pain (generalized vulvodynia). Patients may describe a history of deeper pelvic pain and demonstrate pelvic floor muscle abnormalities on internal exam. It is important to systemically document the findings on physical exam to establish a diagnosis and follow progress once treatment is initiated.

Laboratory investigations can help identify patients with endocrine abnormalities (thyroid stimulating hormone, prolactin) and those with hormonal deficiencies (estradiol (E2), total testosterone (TT), sex hormone binding globulin (SHBG), and calculated free testosterone (cFT)). It is recommended to measure the patient's TT and to use a standardized calculation to calculate the patient's cFT - the amount of testosterone that is actually free to exert physiologic effects. ${ }^{10}$ Some women may demonstrate a low cFT that can contribute to sexual pain, low libido, low arousal and orgasmic dysfunction. ${ }^{11}$ In women on hormonal contraception elevations of SHBG (that tightly bind to testosterone and render it biological inactive) and decreased ovarian production of testosterone can lead to significantly deficient levels of cFT despite normal TT values. The vulvar vestibule is rich in androgen receptors and some patients may be at a higher genetic risk from experiencing pain related to low levels of testosterone. ${ }^{12}$ Symptoms of vulvovaginal irritation in the context of low hormones states are commonly referred to as 
hormonally mediated vulvodynia. Menopausal women may experience similar symptoms from atrophic vulvovaginitis secondary to a low estrogen state. ${ }^{13}$

\section{Treatment}

Treatment is patient specific and requires a multidisciplinary team. Pelvic PT is often needed to improve pelvic floor muscle dysfunction that may present a primary condition or secondary to prolonged vulvar pain. A 2017 systematic review of 43 studies, including 7 randomized control trials, demonstrated significant reduction in pain and improvement in sexual function with pelvic physiotherapy. $^{14}$

A retrospective review from 2010 demonstrated that 25/26 patients experienced statistically significant improvement when physiotherapy was combined with vaginal and/or rectal suppositories. ${ }^{15}$ Studies are now reporting on improvements from pelvic floor onabotulinumtoxin A therapy. ${ }^{16}$ Most high volume centres collaborate closely with pelvic PT.

Many clinicians address vulvar pain prior to treating pelvic muscle floor dysfunction as superficial vulvar pain may make pelvic PT exceedingly difficult. Pain at the vulva may be related to low hormone states which are commonly associated with oral contraception use or menopause. This can be treated with switching to another form of contraception and the use of vestibular and vaginal hormonal therapy in the form of estrogen or a mixture of estrogen and testosterone. Studies have demonstrated improvements with combination estrogen/testosterone topical therapy. ${ }^{17}$ The recently released North American Menopause Society (NAMS) guideline is a helpful resource for managing patients with genitourinary syndrome of menopause related to low estrogen. ${ }^{13}$ It is important to note that patients with hormone sensitive breast cancer (especially those on hormonal supressing therapies) are a unique cohort that require collaboration between the patient, sexual health provider and oncologist. ${ }^{18}$ Treatment of vulvar pain in hormone sensitive breast cancer patients should begin with non-hormonal therapies. ${ }^{13} \mathrm{~A}$ recent study reported that intravaginal testosterone and estradiol-releasing vaginal rings demonstrated a favourable safety profile in the short-term in women on aromatase inhibitors. ${ }^{19}$ However, there is no robust data to suggest safety of local hormonal therapy in this cohort of patients and both providers and patients need to be aware of the uncertainty and potential risk.

Skin lesions suspicious for cancer or autoimmune diseases should be biopsied for definitive diagnosis. Lichen sclerosis and lichen planus are common vulvar conditions that are amenable to topical therapy. ${ }^{20}$

There are a number of non-hormonal treatments that have demonstrated success in the treatment of vulvodynia. A 2004 case series demonstrated significant benefit in 19 of 32 patients treated with topical lidocaine followed by $0.05 \%$ topical capsaicin. ${ }^{21}$ A 2003 case series of premenopausal women with provoked vulvodynia showed a greater than $50 \%$ decrease in sexual pain in over half of patients treated with $5 \%$ topical lidocaine ointment for $6-8$ weeks. ${ }^{22} \mathrm{~A}$ randomized control trial on 46 patients with a history of breast cancer and low estrogen demonstrated improved pain scores and a resumption of sexual activity in $85 \%$ of patients that applied liquid lidocaine prior to penetration. ${ }^{23}$ 
Refractory and severe cases of vulvodynia can ultimately be managed surgically with the resection of vestibular tissues with posterior vaginal advancement flap (vestibulectomy). A cohort of 31 patients recently demonstrated a $60-70 \%$ reduction in pain scores following vestibulectomy ${ }^{24}$. In a larger cohort of 134 patients treated with vestublectomy, 93\% reported being satisfied or very satisfied. ${ }^{25}$

There has been a recent increase in the marketing of minimally invasive technology aimed at improving vulvovaginal atrophy and associated sexual pain. The cost of these treatments are not currently covered by provincial health plans. A randomized double blinded placebo controlled trial of fractionated $\mathrm{CO} 2$ demonstrated benefits to pain scores, vaginal burning and vaginal dryness. ${ }^{26}$ Satisfaction rates were demonstrated to be above $90 \%$ in a cohort study of patients with vulvovaginal atrophy treated with fractionated $\mathrm{CO} 2 .{ }^{27}$ A recent review demonstrated significant improvements in pain scores and sexual function across six studies with a total of 273 women. ${ }^{28}$ Evidence for vaginal laser treatment is new and its role in patient management is currently unclear. Some advocate for this new technology for women who wish to avoid hormonal therapies. ${ }^{29}$ As studies continue to report outcomes and adverse events associated with treatment, patients will be able to weight the risks and benefits of these new treatments in the management of sexual pain and vulvovaginal atrophy.

\section{Low desire}

\section{Evaluation}

Women with low desire will experience an absence or reduction in sexual fantasies and desire for sexual activity that causes distress. Sexual desire is fluid throughout a lifetime however at various points in one's life low sexual desire may cause significant distress. Like painful sex, evaluating and treating women with low desire requires a multidisciplinary approach that includes medical and psychosocial assessments.

A thorough medical history that focuses on the psychosocial factors that may impact one's sexual desire is critical. A review of relevant medications is important as certain therapies may cause decreased sexual desire (table 2). Hormonal contraception has been linked to low desire. ${ }^{30}$ Hormonal assessment should include an E2 level, SHBG, TT, cFT, prolactin and TSH. It is important to try to ascertain if the low desire is acquired or lifelong, and whether it is situational or generalizable (table 3 ) as some treatment options, mentioned below, will depend on this distinction.

\section{Treatment}

A multidisciplinary approach to low desire is important. There are a number of medical treatments with evidence to support their use in patients with low desire. In the United States, Flibanserin (Addyi ${ }^{\circledR}$ ) has been approved for the treatment of low desire in premenopausal women with acquired, generalized low desire. ${ }^{31}$ Studies have also demonstrated efficacy and 
safety in post-menopausal women as well although this still constitutes off label usage. ${ }^{32}$ There is also evidence for the use of central nervous system medications for their pro-dopamine effects as a treatment for patients with sexual side effects from SSRI. ${ }^{33}$ High dose bupropion (150 mg twice daily) has been shown to be an effective treatment for SSRI-related sexual dysfunction. ${ }^{34}$

The Endocrine Society recommends a trial of T therapy for three to six months in postmenopausal women with low androgen levels that are comfortable with off-label use and close monitoring. ${ }^{35}$ A 2008 double blinded randomized control trial in 814 women demonstrated that systemic testosterone therapy improved desire and reduced distress in women with low desire. ${ }^{36}$ A 2017 meta-analysis of post-menopausal women with low desire treated with systemic testosterone ( $n=3035)$ demonstrated statistically significant improvements in sexual desire, orgasm and sexually satisfying events. ${ }^{37}$ The most recent ISSWSH consensus panel suggests that post-menopausal women with low desire may benefit from a trial of systemic testosterone. ${ }^{38}$ Despite the evidence of testosterone therapy's efficacy and short-term safety it is important to note that testosterone treatment is off-label and that there are currently no pharmaceutical formulations made for female doses or application.

\section{Low arousal}

\section{Evaluation}

A number of women will present to their healthcare providers with symptoms related to low arousal that may manifest as a decrease in vaginal lubrication and a decrease in genital warmth related to blood flow. As above, a full medical and sexual history and physical examination is critical. Comorbid conditions that may impact arousal and should be documented as well as a complete list of medications. Hypertension, hyperlipidemia and diabetes have all been linked to FSD and low arousal states. ${ }^{39,40}$ A multidisplinary team consisting of a medical provider, psychosocial support and pelvic PT can all contribute to the evaluation of a patient with low arousal.

\section{Treatment}

The management of comorbid conditions such as diabetes and hypertension should be optimized. ${ }^{41}$ Offending medications may be dose reduced and/or replaced with less sexually inhibiting alternatives if possible. There have been mixed evidence that phosphodiesterase inhibitors may benefit women with poor arousal (by improving pelvic blood flow) and a trial of on-demand PDE5 may be of benefit for some women. ${ }^{42}$ A 2016 randomized control trial of 86 women favoured cognitive behavioral therapy over PDEI usage for the treatment of low arousal. ${ }^{43}$ A randomized double blind study of 34 patients from 2003 failed to show any significant benefits from oral sildenafil in women with low arousal ${ }^{44}$. The conflicting evidence on the role of PDEI in women with low arousal appears to suggest that the patients with arousal disorder are a heterogenous group of patients and some may benefit from PDEI. 


\section{Orgasmic dysfunction}

\section{Evaluation}

For women complaining of distress related to delayed or absent orgasms it is necessarily to take a detailed medical history, psychosocial history and identify possible offending medications. Understanding if the problem is lifelong or acquired and distinguishing between completely absent orgasms and delayed or less intense orgasms are important points in the sexual history that will guide the medical and psychosocial treatment. Similar to the approach to low libido clinicians must also identify if the problem is situational or acquired. All of these distinctions that are elucidated on a thorough sexual history aid both the psychosocial and medical treatment of orgasmic dysfunction. Orgasmic dysfunction is often associated with other forms of sexual dysfunction- for example patients with sexual pain and poor arousal will often find it difficult to reach orgasm. The evaluation should therefore put the orgasmic dysfunction in context of an individual's broader sexual function. Physical exam including vulvoscopy should include an assessment of the clitoris. Some women may exhibit phimosis (covering) of the clitoris with or without an underlying etiology (such as Lichen Sclerosis). Patients should also be screened, and examined for, female genital mutilation which often involves damaging the external portion of the clitoris.

\section{Treatment}

The medical treatment of orgasmic problems is challenging although there has been reports of success with mindfulness, yoga, the use of sex toys and sex therapy. ${ }^{45}$ Directed masturbation has demonstrated efficacy for women with lifelong anorgasmia. ${ }^{46}$ As previously noted SSRI have been linked to delayed or absent orgasms and can be dose reduced, replaced with other psychiatric medications or combined with buproprion. Some clinicians have experimented with the off label use of testosterone, dopamine agonists and yohimbine hydrochloride with encouraging results although there are no clinical trials to currently support their usage. A randomized control trial published in the Journal of the American Medical Association demonstrated that sildenafil improved orgasm is women on SSRIs better than placebo. ${ }^{47}$ Small cohort studies have shown high patient satisfaction rates in patients with clitoral phimosis underoing surgery or CO2 laser treatment ${ }^{48,49}$.

\section{Conclusion}

There is a strong basis for the evidenced based evaluation and management of FSD. A basic understanding of the common presentations, evaluation and medical management of FSD can empower primary care providers to inquire about sexual symptoms and offer an appropriate workup and initial treatment (see table 4). Sharing a common approach to FSD allows easier communication among patients and between healthcare providers. 


\section{References}

1. Goldstein I, Lue TF, Padma-Nathan H, Rosen RC, Steers WD, Wicker PA. Oral Sildenafil in the Treatment of Erectile Dysfunction. J Urol. 1998;160(5):1944-1945.

doi:10.1016/S0022-5347(01)62474-1.

2. Sobecki JN, Curlin FA, Rasinski KA, Lindau ST. What we don"t talk about when we don”t talk about sex: results of a national survey of U.S. obstetrician/gynecologists. J Sex Med. 2012;9(5):1285-1294. doi:10.1111/j.1743-6109.2012.02702.x.

3. ISSWSH/NAMS Consensus Committee. ISSWSH position statement. J Sex Med. 2014;11(5):1109. doi:10.1111/jsm.12541.

4. Leon-Carlyle M, Schmocker S, Victor JC, et al. Prevalence of Physiologic Sexual Dysfunction Is High Following Treatment for Rectal Cancer: But Is It the Only Thing That Matters? Dis Colon Rectum. 2015;58(8):736-742. doi:10.1097/DCR.0000000000000409.

5. DeGregorio MW, Zerbe RL, Wurz GT. Ospemifene: a first-in-class, non-hormonal selective estrogen receptor modulator approved for the treatment of dyspareunia associated with vulvar and vaginal atrophy. Steroids. 2014;90:82-93.

doi:10.1016/j.steroids.2014.07.012.

6. $\quad$ Kaufman MB. Pharmaceutical Approval Update. P T. 2017;42(2):90-91.

7. Puppo G, Puppo V. U.S. Food and Drug Administration Approval of Addyi (Flibanserin) for Treatment of Hypoactive Sexual Desire Disorder. European Urology. 2016;69(2):379380. doi:10.1016/j.eururo.2015.09.050.

8. Latif EZ, Diamond MP. Arriving at the diagnosis of female sexual dysfunction. Fertil Steril. 2013;100(4):898-904. doi:10.1016/j.fertnstert.2013.08.006.

9. Stephenson KR, Toorabally N, Lyons L, M Meston C. Further Validation of the Female Sexual Function Index: Specificity and Associations With Clinical Interview Data. J Sex Marital Ther. 2016;42(5):448-461. doi:10.1080/0092623X.2015.1061078.

10. Vermeulen A, Verdonck L, Kaufman JM. A critical evaluation of simple methods for the estimation of free testosterone in serum. J Clin Endocrinol Metab. 1999;84(10):36663672. doi:10.1210/jcem.84.10.6079.

11. Vale FBC, Coimbra BB, Lopes GP, Geber S. Sexual dysfunction in premenopausal women could be related to hormonal profile. Gynecol Endocrinol. 2017;33(2):145-147. doi:10.1080/09513590.2016.1226793.

12. Goldstein AT, Belkin ZR, Krapf JM, et al. Polymorphisms of the androgen receptor gene and hormonal contraceptive induced provoked vestibulodynia. J Sex Med. 2014;11(11):2764-2771. doi:10.1111/jsm.12668.

13. The 2017 hormone therapy position statement of The North American Menopause Society. Menopause. 2017;24(7):728-753. doi:10.1097/GME.0000000000000921. 
14. Morin M, Carroll M-S, Bergeron S. Systematic Review of the Effectiveness of Physical Therapy Modalities in Women With Provoked Vestibulodynia. Sex Med Rev. 2017;5(3):295-322. doi:10.1016/j.sxmr.2017.02.003.

15. Rogalski MJ, Kellogg-Spadt S, Hoffmann AR, Fariello JY, Whitmore KE. Retrospective chart review of vaginal diazepam suppository use in high-tone pelvic floor dysfunction. Int Urogynecol J. 2010;21(7):895-899. doi:10.1007/s00192-009-1075-7.

16. Pacik PT, Geletta S. Vaginismus Treatment: Clinical Trials Follow Up 241 Patients. Sex Med. 2017;5(2):e114-e123. doi:10.1016/j.esxm.2017.02.002.

17. Burrows LJ, Goldstein AT. The treatment of vestibulodynia with topical estradiol and testosterone. Sex Med. 2013;1(1):30-33. doi:10.1002/sm2.4.

18. American College of Obstetricians and Gynecologists' Committee on Gynecologic Practice, Farrell R. ACOG Committee Opinion No. 659: The Use of Vaginal Estrogen in Women With a History of Estrogen-Dependent Breast Cancer. Obstet Gynecol. 2016;127(3):e93-e96. doi:10.1097/AOG.0000000000001351.

19. Melisko ME, Goldman ME, Hwang J, et al. Vaginal Testosterone Cream vs Estradiol Vaginal Ring for Vaginal Dryness or Decreased Libido in Women Receiving Aromatase Inhibitors for Early-Stage Breast Cancer: A Randomized Clinical Trial. JAMA Oncol. 2017;3(3):313-319. doi:10.1001/jamaoncol.2016.3904.

20. Burrows LJ, Creasey A, Goldstein AT. The treatment of vulvar lichen sclerosus and female sexual dysfunction. J Sex Med. 2011;8(1):219-222. doi:10.1111/j.17436109.2010.02077.x.

21. Murina F, Radici G, Bianco V. Capsaicin and the treatment of vulvar vestibulitis syndrome: a valuable alternative? MedGenMed. 2004;6(4):48.

22. Zolnoun DA, Hartmann KE, Steege JF. Overnight $5 \%$ lidocaine ointment for treatment of vulvar vestibulitis. Obstet Gynecol. 2003;102(1):84-87.

23. Goetsch MF, Lim JY, Caughey AB. A Practical Solution for Dyspareunia in Breast Cancer Survivors: A Randomized Controlled Trial. J Clin Oncol. 2015;33(30):3394-3400. doi:10.1200/JCO.2014.60.7366.

24. Kliethermes CJ, Shah M, Hoffstetter S, Gavard JA, Steele A. Effect of Vestibulectomy for Intractable Vulvodynia. J Minim Invasive Gynecol. 2016;23(7):1152-1157. doi:10.1016/j.jmig.2016.08.822.

25. Goldstein AT, Klingman D, Christopher K, Johnson C, Marinoff SC. Surgical treatment of vulvar vestibulitis syndrome: outcome assessment derived from a postoperative questionnaire. J Sex Med. 2006;3(5):923-931. doi:10.1111/j.1743-6109.2006.00303.x.

26. Cruz VL, Steiner ML, Pompei LM, et al. Randomized, double-blind, placebo-controlled clinical trial for evaluating the efficacy of fractional CO2 laser compared with topical estriol in the treatment of vaginal atrophy in postmenopausal women. Menopause. July 2017:1. doi:10.1097/GME.0000000000000955.

27. Sokol ER, Karram MM. Use of a novel fractional CO2 laser for the treatment of 
genitourinary syndrome of menopause: 1-year outcomes. Menopause. 2017;24(7):810814. doi:10.1097/GME.0000000000000839.

28. Salvatore S, Pitsouni E, Del Deo F, Parma M, Athanasiou S, Candiani M. Sexual Function in Women Suffering From Genitourinary Syndrome of Menopause Treated With Fractionated CO2 Laser. Sex Med Rev. 2017;5(4):486-494.

doi:10.1016/j.sxmr.2017.07.003.

29. Pieralli A, Fallani MG, Becorpi A, et al. Fractional CO2 laser for vulvovaginal atrophy (VVA) dyspareunia relief in breast cancer survivors. Arch Gynecol Obstet. 2016;294(4):841-846. doi:10.1007/s00404-016-4118-6.

30. Malmborg A, Persson E, Brynhildsen J, Hammar M. Hormonal contraception and sexual desire: A questionnaire-based study of young Swedish women. Eur J Contracept Reprod Health Care. 2016;21(2):158-167. doi:10.3109/13625187.2015.1079609.

31. Derogatis LR, Komer L, Katz M, et al. Treatment of hypoactive sexual desire disorder in premenopausal women: efficacy of flibanserin in the VIOLET Study. J Sex Med. 2012;9(4):1074-1085. doi:10.1111/j.1743-6109.2011.02626.x.

32. Simon JA, Kingsberg SA, Shumel B, Hanes V, Garcia M, Sand M. Efficacy and safety of flibanserin in postmenopausal women with hypoactive sexual desire disorder: results of the SNOWDROP trial. Menopause. 2014;21(6):633-640. doi:10.1097/GME.0000000000000134.

33. Lodise NM. Hypoactive sexual desire disorder in women: treatment options beyond testosterone and approaches to communicating with patients on sexual health. Pharmacotherapy. 2013;33(4):411-421. doi:10.1002/phar.1209.

34. Taylor MJ, Rudkin L, Bullemor-Day P, Lubin J, Chukwujekwu C, Hawton K. Strategies for managing sexual dysfunction induced by antidepressant medication. Taylor MJ, ed. Cochrane Database Syst Rev. 2013;22(5):CD003382. doi:10.1002/14651858.CD003382.pub3.

35. Wierman ME, Arlt W, Basson R, et al. Androgen therapy in women: a reappraisal: an Endocrine Society clinical practice guideline. In: Vol 99. 2014:3489-3510. doi:10.1210/jc.2014-2260.

36. Davis SR, Moreau M, Kroll R, et al. Testosterone for low libido in postmenopausal women not taking estrogen. N Engl J Med. 2008;359(19):2005-2017. doi:10.1056/NEJMoa0707302.

37. Achilli C, Pundir J, Ramanathan P, Sabatini L, Hamoda H, Panay N. Efficacy and safety of transdermal testosterone in postmenopausal women with hypoactive sexual desire disorder: a systematic review and meta-analysis. Fertil Steril. 2017;107(2):475-482.e15. doi:10.1016/j.fertnstert.2016.10.028.

38. Goldstein I, Kim NN, Clayton AH, et al. Hypoactive Sexual Desire Disorder: International Society for the Study of Women's Sexual Health (ISSWSH) Expert Consensus Panel Review. Mayo Clin Proc. 2017;92(1):114-128. doi:10.1016/j.mayocp.2016.09.018. 
39. Cortelazzi D, Marconi A, Guazzi M, et al. Sexual dysfunction in pre-menopausal diabetic women: clinical, metabolic, psychological, cardiovascular, and neurophysiologic correlates. Acta Diabetol. 2013;50(6):911-917. doi:10.1007/s00592-013-0482-x.

40. Pontiroli AE, Cortelazzi D, Morabito A. Female sexual dysfunction and diabetes: a systematic review and meta-analysis. J Sex Med. 2013;10(4):1044-1051.

doi:10.1111/jsm.12065.

41. Krysiak R, Drosdzol-Cop A, Skrzypulec-Plinta V, Okopień B. Sexual Functioning and Depressive Symptoms in Women with Diabetes and Prediabetes Receiving Metformin Therapy: A Pilot Study. Exp Clin Endocrinol Diabetes. 2017;125(1):42-48. doi:10.1055/s0042-116594.

42. Chivers ML, Rosen RC. Phosphodiesterase type 5 inhibitors and female sexual response: faulty protocols or paradigms? J Sex Med. 2010;7(2 Pt 2):858-872. doi:10.1111/j.17436109.2009.01599.x.

43. Omidi A, Ahmadvand A, Najarzadegan MR, Mehrzad F. Comparing the effects of treatment with sildenafil and cognitive-behavioral therapy on treatment of sexual dysfunction in women: a randomized controlled clinical trial. Electron Physician. 2016;8(5):2315-2324. doi:10.19082/2315.

44. Basson R, Brotto LA. Sexual psychophysiology and effects of sildenafil citrate in oestrogenised women with acquired genital arousal disorder and impaired orgasm: a randomised controlled trial. BJOG. 2003;110(11):1014-1024.

45. Hucker A, McCabe MP. Incorporating Mindfulness and Chat Groups Into an Online Cognitive Behavioral Therapy for Mixed Female Sexual Problems. J Sex Res. 2015;52(6):627-639. doi:10.1080/00224499.2014.888388.

46. Meston CM, Hull E, Levin RJ, Sipski M. Disorders of orgasm in women. J Sex Med. 2004;1(1):66-68. doi:10.1111/j.1743-6109.2004.10110.x.

47. Nurnberg HG, Hensley PL, Heiman JR, Croft HA, Debattista C, Paine S. Sildenafil treatment of women with antidepressant-associated sexual dysfunction: a randomized controlled trial. JAMA. 2008;300(4):395-404. doi:10.1001/jama.300.4.395.

48. Kroft J, Shier M. A novel approach to the surgical management of clitoral phimosis. $J$ Obstet Gynaecol Can. 2012;34(5):465-471.

49. Goldstein AT, Burrows LJ. Surgical treatment of clitoral phimosis caused by lichen sclerosus. Am J Obstet Gynecol. 2007;196(2):126.e1-.e4. doi:10.1016/j.ajog.2006.08.023. 
Figures and Tables

\begin{tabular}{|l|l|}
\hline \multicolumn{2}{|l|}{ Table 1. Initial approach to patients with female sexual dysfunction } \\
\hline History & History of presenting illness \\
& Sexual history \\
& Complete gynecological history \\
& Birth control and hormone therapy history \\
& Medications \\
& Psych history \\
& Standardized Screener (e.g., FSFI) \\
\hline Physical Exam & General appearance \\
& Abdominal exam \\
& Vulvoscopy \\
& Presence of dermatological lesions ( \pm biopsy) \\
& Cue tip testing for vulvodynia \\
& Vaginal pH for atrophic vaginitis \\
& Microscopy/KOH testing for clue cells, parabasal cells \\
\hline Investigations & TSH, prolactin \\
& Sex hormone-binding globulin \\
\hline & Estradiol \\
& Total testosterone \\
& Calculated free testosterone \\
\hline
\end{tabular}

FSFI: Female Sexual Function Inventory.

\begin{tabular}{|l|l|}
\hline Table 2. Medications impacting female sexual function \\
\hline Class & Examples \\
\hline Psych/Neuro & Selective serotonin reuptake inhibitors \\
& Benzodiazepines \\
& Lithium \\
& Antipsychotics \\
& Phenytoin \\
\hline Hormonal & Contraception \\
& Anti-androgens \\
& Tamoxifen \\
& Aromatase inhibitors \\
& Ketoconazole \\
\hline Pain & Opioids \\
\hline
\end{tabular}




\begin{tabular}{|l|l|}
\hline & Tricyclic antidepressants \\
& Indomethacin \\
\hline Cardiovascular & Spironolactone \\
& Digoxin \\
& Methyldopa \\
& Beta blockers \\
& Clonidine \\
\hline
\end{tabular}

\begin{tabular}{|l|l|}
\hline \multicolumn{2}{|l|}{ Table 3. Classification of low sexual desire } \\
\hline Menopausal status & Premenopausal vs. postmenopausal \\
\hline Onset & Lifelong vs. acquired \\
\hline Situation & Situational vs. generalized \\
\hline
\end{tabular}

\begin{tabular}{|l|l|}
\hline Table 4. Summary of diagnosis and treatment of female sexual dysfunction \\
\hline Diagnosis & Treatment \\
\hline Sexual pain & Local hormone therapy \\
& Counselling \\
& Pelvic PT \\
Vaginal/rectal suppositories & Topical lidocaine \\
Capsaicin & Vestibulectomy \\
\hline Low desire & $\begin{array}{l}\text { Hormonal therapy } \\
\text { Counselling } \\
\text { Buproprion } \\
\text { Flibanserin (not available in Canada) }\end{array}$ \\
\hline Low arousal & $\begin{array}{l}\text { Hormonal therapy } \\
\text { Counselling } \\
\text { PDE5 inhibitors (e.g., sildenafil) }\end{array}$ \\
\hline Orgasmic dysfunction & $\begin{array}{l}\text { Mindfulness, sex therapy } \\
\text { Hormonal therapy } \\
\text { Buprioprion } \\
\text { PDE inhibitors (e.g., sildenafil) } \\
\text { Yohimbine hydrocholoride }\end{array}$ \\
\hline
\end{tabular}

PDE: phosphodiesterase; PT: physical therapy. 